\title{
Electroacupuncture Inhibits Spinal Interleukin-17A to Alleviate Inflammatory Pain in a Rat Model
}

\author{
Xianze Meng ${ }^{1,2}$, Lixing Lao ${ }^{1}$, Xue-Yong Shen ${ }^{3}$, Brian M. Berman ${ }^{1}$, Ke Ren ${ }^{4}$, Pin-Kang Wei ${ }^{2}$ and \\ Rui-Xin Zhang ${ }^{1, *}$
}

${ }^{l}$ Center for Integrative Medicine, School of Medicine, University of Maryland, Baltimore, MD 21201, USA; ${ }^{2}$ Department of Traditional Chinese Medicine, Shanghai Changzheng Hospital, Second Military Medical University, Shanghai, 200001, P. R. China; ${ }^{3}$ Acupuncture College, Shanghai University of Traditional Chinese Medicine, Shanghai 201203 P. R. China; ${ }^{4}$ Department of Neural and Pain Sciences, Dental School, University of Maryland, Baltimore, MD 21201, USA

\begin{abstract}
Although acupuncture analgesia has been reported in clinical trials, its mechanisms have been unclear. It was recently reported that spinal astrocytes-produced interleukin-17A (IL-17A) facilitates inflammatory pain. Hypothesizing that electroacupuncture (EA) would suppress inflammation-enhanced IL-17A synthesis to inhibit pain, we induced hyperalgesia, as measured by decreased paw withdrawal latency (PWL) to a noxious thermal stimulus, by subcutaneously injecting complete Freund's adjuvant (CFA, $0.08 \mathrm{ml}, 40 \mu \mathrm{g}$ Mycobacterium tuberculosis) into the hind paws of rats, or intrathecal (i.t.) IL-17A (400 ng in $10 \mu \mathrm{l}$ ) into the lumbar spinal cord. We then gave EA at acupoint GB30 for two 20-min periods, once immediately after CFA or IL-17A administration and again $2 \mathrm{~h}$ post-injection. For sham control, EA needles were inserted into GB30 without stimulation. PWL was measured before and 2.5 and $24 \mathrm{~h}$ after injection. Spinal IL-17A, IL-17 receptor A (IL-17RA), and phosphorylated NR1, an essential subunit of the N-methyl D-aspartate receptor (NMDAR), were determined $24 \mathrm{~h}$ post-CFA or -IL-17A using immunohistochemistry and western blot. Compared to sham control, EA inhibited CFA-caused thermal hyperalgesia 2.5 and $24 \mathrm{~h}$ post-CFA and concurrently suppressed inflammation-enhanced IL-17A and IL-17RA synthesis and NR1 phosphorylation in the ipsilateral spinal cord. EA inhibited IL-17A-produced thermal hyperalgesia, IL-17RA synthesis and NR1 phosphorylation. Our data suggest that EA inhibits inflammatory pain by blocking spinal IL-17A synthesis. Since previous study shows that IL-17A is located in astrocytes and IL-17RA and NR1 are in neurons, the data suggest that EA alleviates pain by modulating glia-neuronal interactions that involve IL-17A, IL-17RA, and NR1 phosphorylation.
\end{abstract}

Keywords: Hyperalgesia, Pain, Spinal cord, Interleukin-17A, NMDA, Electroacupuncture, Astrocytes.

\section{INTRODUCTION}

Spinal glia is known to be involved in transmission and modulation of noxious messages. These cells produces a number of algesic substances such as the proinflammatory cytokines interleukin 1 beta (IL-1 $\beta$ ), tumor necrosis factor alpha (TNF- $\alpha$ ), and IL-6 to promote spinal transmission and processing of noxious signals $[1,2]$. It is reported that IL- $1 \beta$ is up-regulated in spinal astrocytes to increase NMDA NR1 phosphorylation, an essential subunit of the N-methyl Daspartate receptor (NMDAR), which in turn promote pain in an inflammatory pain rat model [3-6]. IL-17A, recently found in astrocytes of patients with multiple sclerosis [7], increase in concentration in the spinal cord of rats with nerve injury [8]. Further, intrathecal (i.t.) injection of IL-17A in rats induced thermal hyperalgesia and peripheral inflammation caused up-regulation of IL-17A in spinal astrocytes and IL-17 receptor A (IL-17RA) in spinal neurons [9]. These studies indicate that spinal IL-17A facilitates pain.

*Address correspondence to this author at the Center for Integrative Medicine, 685 W. Baltimore Street, MSTF Rm 8-22, Baltimore, MD 21201, USA; Tel: 410-706-1582; Fax: 410-706-1583;

E-mail: Rzhan001@umaryland.edu
Acupuncture has been used in China and other Asian countries for thousands of years and is an option for managing pain [10]. Although acupuncture analgesia has been reported in clinical trials [11], its mechanisms are still unclear. Previous study shows that electroacupuncture (EA) significantly increases paw withdrawal latency (PWL) and inhibits intra-plantar CFA-induced up-regulation of glial fibrillary acidic protein, a marker of astrocytes [12]. EA also markedly inhibits intra-articular CFA-induced up-regulation of IL-1 $\beta$, IL-6, and TNF $\alpha$ in the spinal cord [13-15].

Such studies suggest that EA modulates astrocyte activity to inhibit pain, but whether the modality regulates IL-17A synthesis to alleviate inflammatory pain was not known. We hypothesized that EA down-regulates inflammationexaggerated spinal IL-17A and its receptors to inhibit inflammatory pain. Since IL-17A facilitates NR1 phosphorylation [9], we also hypothesized that EA alleviates pain by inhibiting NR1 phosphorylation. These hypotheses were tested in a rat model of inflammatory pain.

\section{MATERIALS AND METHODOLOGY} Animal Preparation

Male Sprague-Dawley rats weighing 270-300 g (Harlan, Indianapolis, IN) were housed under controlled laboratory 
conditions. Room temperature was $22^{\circ} \mathrm{C}$ and humidity was $40 \%-60 \%$, with 12-h light-dark cycles, food and water ad libitum. They were acclimatized to the environment for five days prior to experimentation. The animal protocols were approved by the Institutional Animal Care and Use Committee of the University of Maryland School of Medicine, Maryland, USA.

\section{Experimental Design}

Three experiments were carried out to study possible EA mechanisms on and modulation of 1) intra-plantar CFAcaused thermal hyperalgesia in three groups: CFA + EA, $\mathrm{CFA}+$ sham and saline + sham, and i.t. IL-17A-caused thermal hyperalgesia in three groups: IL-17A + EA, IL-17A + sham, and saline (i.t.) + sham, 2) IL-17A during CFAinduced inflammation, and 3) spinal IL-17RA and phosphorylated NR1 (p-NR1).

\section{I.t. IL-17A Delivery}

Spinal punctures were conducted as previously stated [9]. Briefly, under isoflurane anesthesia, the rat's dorsal pelvic area was disinfected with $70 \%$ alcohol. A 21-gauge sterile needle was inserted between lumbar vertebrae L5 and L6. A catheter of a PE10 polyethylene tube (Clay Adams, USA), which was pre-filled with $10 \mu \mathrm{l}$ of $400 \mathrm{ng}$ IL-17A (ProSpec, Israel) or vehicle isolated from $5 \mu \mathrm{l}$ of saline using a small air bubble, was placed into the needle and advanced $4 \mathrm{~cm}$ from the tip of the needle up to the lumber enlargement, which was confirmed by a tail-twitch. The drug, or vehicle, was slowly injected and flushed with saline. Three minutes later, the catheter and the needle were respectively removed.

\section{Induction of Hind Paw Hyperalgesia}

CFA (Sigma, USA; $0.08 \mathrm{ml}, 40 \mu \mathrm{g}$ Mycobacterium tuberculosis), which was suspended in an 1:1 oil/saline emulsion, was injected into the plantar surface of one hind paw of the rat to result in inflammatory hyperalgesia [16]. The inflammation appeared as redness, edema, and hyperresponsiveness to noxious stimuli, and was limited to the injected paw. Hyperalgesia was assessed with PWL to a noxious heat stimulus.

\section{Paw Withdrawal Latency}

The rats in experiments 1 were tested for hind paw withdrawal latency (PWL) by a previously described method $[16,17]$. They were acclimatized for $30 \mathrm{~min}$ under an inverted clear plastic box on the glass surface of a Paw Thermal Stimulator System (UCSD, San Diego, USA). The plantar surface of each hind paw was stimulated with a radiant heat stimulus from lamp bulb (CXL/CXR, $8 \mathrm{~V}, 50$ W) underneath the glass floor. Four tests were performed with 5-min interval between each test. When hindpaw was moved away from the stimulus, PWL to the nearest $0.1 \mathrm{~s}$ was automatically recorded. Baseline PWL was approximately $10.0 \mathrm{~s}$ in naive animals. Paws were stimulated randomly to exclude order effects. A 20 -s cut-off was set to avoid tissue damage.

Mean PWL was determined by calculating average of the latency of four tests. The investigator who conducted the PWL tests was blind to animal group.

\section{Electroacupuncture}

Electroacupuncture (EA) was given at acupoint GB30 as described before [19]. In humans, GB30 is found at the junction of the lateral $1 / 3$ and medial $2 / 3$ of the distance between the greater trochanter and the sacral hiatus. Equivalent anatomical landmarks were used to locate these points in the rat. The transposition of an acupoint from the known human map to the anatomically comparable position in animals is widely used to determine points in animals [1821] and has been demonstrated to be effective [19, 22, 23]. After cleaning the skin with alcohol swabs, one investigator swiftly inserted two \#32 gauge, 0.5" acupuncture needles bilaterally into each hind limb of the rat at GB 30 while another gently held the animal. The needles were stabilized with adhesive tape [19]. EA $(10 \mathrm{~Hz}, 2 \mathrm{~mA}, 0.4 \mathrm{~ms}$ pulse width) was delivered by a stimulator (Electrostimulator 8-C, Pantheon Research Inc) via two electrodes for two 20-min periods, once immediately after CFA or IL-17A administration and again $2 \mathrm{~h}$ post-CFA or post-IL-17A. A symmetrical biphasic wave was delivered to each electrode so that it was alternately positive and negative to stimulate the bilateral needles. To minimize discomfort, stimulation intensity was gradually increased over a period of $2 \mathrm{~min}$ to $2 \mathrm{~mA}$, which we have found to be the maximum level that can be tolerated by unrestrained rats [24]. During EA treatment, each rat was placed under an inverted clear plastic chamber approximately $5 " \times 8 " \times 11$ " and neither restrained nor given any anesthetic. Mild muscle twitching was observed. The animals remained awake and still during treatment and gave no observable signs of distress.

For sham control, acupuncture needles were inserted bilaterally into GB30 without electrical or manual needle manipulation. Sham EA showed little anti-hyperalgesia in our previous study [19], which makes it an appropriate control for non-specific needling effects. Sham- and EAtreated animals were handled identically. The investigators performing the behavioral tests were blind to treatment assignments.

\section{Immunoflurescence}

Rats ( $n=3$ / group) were deeply anesthetized $24 \mathrm{hr}$ postCFA with sodium pentobarbital $[60 \mathrm{mg} / \mathrm{kg}$, intraperitoneal injection (i.p.)] and immediately perfused transcardially using 4\% paraformaldehyde (Sigma) in $0.1 \mathrm{M}$ phosphate buffer $(\mathrm{PB})$ at $\mathrm{pH}$ 7.4. The lumbar 4-5 spinal cord was harvested, submerged in the fixative for another $2 \mathrm{~h}$ at $4{ }^{\circ} \mathrm{C}$, and immersed in $30 \%$ sucrose $(\mathrm{w} / \mathrm{v})$ in $\mathrm{PB}$ saline $(\mathrm{PBS})$ overnight for cryoprotection. The tissue was sectioned at $30-$ $\mu \mathrm{m}$. After rinsed in PBS and blocked in PBS containing $10 \%$ normal donkey serum for $60 \mathrm{~min}$, sections were incubated overnight at room temperature with rabbit polyclonal IL-17A antibody (1:50, Cat\# sc-7927, Santa Cruz Biotechnology, Inc.). After three washings in PBS each for $10 \mathrm{~min}$, the sections were incubated in CY2-conjugated donkey anti-rabbit (1:200, Jackson ImmunoResearch Laboratories). Control sections were similarly processed but with no primary antisera. The sections were placed on gelatin-coated slides, covered with aqueous mounting medium (Biomeda Corp., CA), and observed with a Nikon fluorescence microscope. IL-17A-immunopositive cells were magnified with a $20 \times$ objective lens and counted on 10 
randomly-selected sections from each rat. Mean of IL-17Aimmunopositive cell number on each section were determined for each rat and then for the group.

\section{Western Blot}

The rats ( $\mathrm{n}=4$ /group) were anesthetized with sodium pentobarbital $(60 \mathrm{mg} / \mathrm{kg}$, i.p.) and decapitated $24 \mathrm{~h}$ post-CFA or -IL-17A. After a high-pressure injection of saline into the sacral spinal cord, the ipsilateral and contralateral dorsal portions of the lumbar 4-5 spinal cord were harvested. The ipsilateral spinal dorsal horn was homogenized in protein extraction buffer containing 1\% EDTA and 1\% Halt $^{\mathrm{TM}}$ Protease and Phosphatase Inhibitor Cocktail (Thermo Scientific, USA) and centrifuged at $14000 \mathrm{rpm}$ for $10 \mathrm{~min}$ at $4{ }^{\circ} \mathrm{C}$. The upper portion was collected and measured with the Bio-Rad Protein Assay for protein concentration. The same quantity of protein was mixed with loading buffer, and electrophoresed on a 4-20\% (w/v) SDS-PAGE and transferred onto a polyvinylidine difluoride (PVDF) membrane (Bio-Rad, USA) with a Trans-Blot Cell System (Bio-Rad). The membrane was blocked for $1 \mathrm{~h}$ at room temperature with $5 \%(\mathrm{w} / \mathrm{v})$ milk in TBS $(20 \mathrm{mM}$ Tris, 150 $\mathrm{mM} \mathrm{NaCl}, \mathrm{pH}$ 7.4) containing $0.1 \%$ Tween 20 (TBST). The blocked membrane was then probed overnight at $4^{\circ} \mathrm{C}$ with phosphor-NR1 antiserum (Serine 896, 1:500, Cat \# sc31669-R, Santa Cruz) or IL-17RA antiserum (1:500, Cat \# sc-30175, Santa Cruz). After washed with TBST buffer, the membrane was incubated for $2 \mathrm{~h}$ at room temperature with goat anti-rabbit horseradish peroxidase-conjugated $\mathrm{IgG}$ (1:2000; KPL) diluted in 5\% milk in TBST buffer. The enhanced chemilluminescence detection system (ECL, Thermo Scientific) was used to detect the immunoreactivity of membrane proteins. Autoradiograms were digitized, and quantified with Scion NIH Image 1.60. The membranes were re-probed with $\beta$-actin antibody (1:5000, Sigma) as a loading control after washed in stripping buffer (Thermo Scientific) for $20 \mathrm{~min}$. The investigators who harvested the spinal cord and carried out the western blot were blinded to the animal assignment.

\section{Data Analysis}

PWL data were shown in mean \pm S.E.M. and analyzed using repeated measures analysis of variance (ANOVA) followed by Tukey's multiple comparisons (GraphPad Prism). Protein data were analyzed with one-way betweensubject ANOVA followed by the Tukey's multiple comparison. The statistical significance level was set at $\mathrm{P}<0.05$.

\section{RESULTS}

\section{EA Alleviated CFA- and IL-17A-Caused Thermal Hyperalgesia}

Baseline PWL to noxious thermal stimuli was similar before CFA in all groups of rats. CFA injection significantly shortened ipsilateral PWL. Saline injection produced no effect on PWL (Fig. 1A). Contralateral PWL had no changes after CFA (data not shown). Repeated-measures ANOVA revealed significant differences among the three groups of rats, respectively given CFA + EA, CFA + sham, or saline + sham at 2 and 24 h post-CFA. Tukey's multiple comparisons revealed that EA significantly $(\mathrm{P}<0.05)$ increased $\mathrm{PWL}$ of the hind paw ipsilateral to the CFA injection compared to sham control (Fig. 1A) but had no significant effect on contralateral hind paw PWL (data not shown).

I.t. IL-17A resulted in significant $(\mathrm{P}<0.05)$ decrease of PWL of bilateral hind paws 2-24 after its injection compared to saline injection, indicating that the IL-17A induced thermal hyperalgesia (Fig. 1B). The mean values of bilateral PWL were presented. EA significantly increased PWL compared to sham control in IL-17A injected rats $(\mathrm{P}<0.05)$,

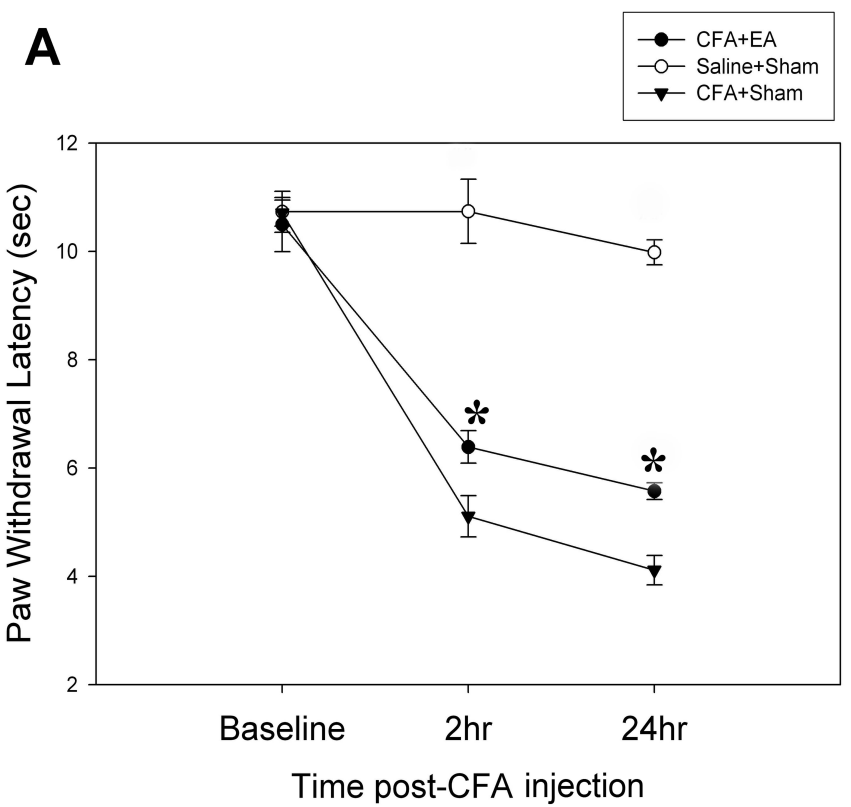

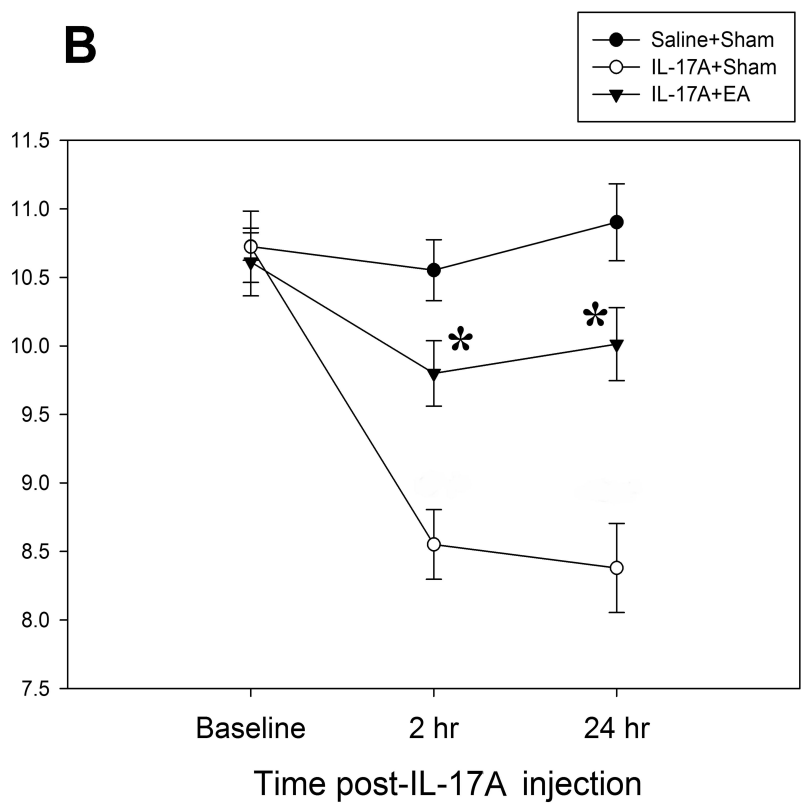

Fig. (1). Effect of EA on PWL in CFA- (A) and IL-17A- (B) injected rats. CFA and IL-17A injections significantly decreased PWL, an indication of thermal hyperalgesia. EA treatment significantly prolonged $\mathrm{PWL}$, indicating $\mathrm{EA}$ analgesia $(\mathrm{N}=7$ per group). * $\mathrm{P}<0.05$ vs. $\mathrm{CFA}$ +sham or IL-17A + sham. 
suggesting that EA attenuates IL-17A-induced thermal hyperalgesia compared to sham.

\section{EA Down-Regulated IL-17A Synthesis in Spinal Dorsal Horn Astrocytes}

IL-17A-immunoreactive astrocytes mainly existed in laminae I-II and V-VI. In ipsilateral laminae I-II, IL-17A immunoreactive cells in CFA + EA rats were significantly more than those in saline control rats but significantly fewer than those in CFA + sham rats, indicating that EA suppressed CFA inflammation-caused IL-17A up-regulation in the dorsal horn. In laminae V-VI, there were significantly more ipsilateral IL-17A immunoreactive cells in rats with CFA injection than in control rats, but there was no significant difference between CFA + EA and CFA + sham rats (Fig. 2).

\section{EA Down-Regulated Dorsal Horn IL-17RA in CFA- and IL-17A-Injected Rats}

Relative spinal IL-17RA levels were significantly $(\mathrm{P}<0.05)$ higher in $\mathrm{CFA}$-injected rats given sham EA treatment than in saline-injected rats. This suggests that peripheral inflammation induced IL-17RA up-regulation. Spinal IL-17RA levels were significantly lower in CFAinjected rats given EA treatment than in CFA-injected rats given sham EA $(\mathrm{P}<0.05)$. This indicates that EA treatment significantly inhibited peripheral inflammation-induced IL17RA up-regulation compared to sham $(\mathrm{P}<0.05$; Fig. 3). Similarly, i.t. IL-17A significantly induced up-regulation of IL-17RA and EA treatment mitigated such up-regulation compared to sham control (Fig. 4).

\section{EA Down-Regulated Dorsal Horn NR1 Phosphorylation in CFA- and IL-17A-Injected Rats}

Spinal p-NR1 was significantly higher in CFA-injected rats given sham EA treatment than in saline-injected rats $(\mathrm{P}<0.05)$. This suggests that peripheral inflammation enhances NR1 phosphorylation. Spinal p-NR1 levels were significantly lower in CFA +EA than CFA+ sham EA rats $(\mathrm{P}<0.05)$, showing that $\mathrm{EA}$ treatment significantly suppressed peripheral inflammation-induced NR1 phosphorylation compared to sham EA ( $<<0.05$; Fig. 5). Consistently, IL-17A significantly enhanced NR1 phosphorylation and EA treatment decreased such phosphorylation (Fig. 6).

\section{DISCUSSION}

In this study, EA significantly alleviated CFAinflammation- and IL-17A-induced thermal hyperalgesia and significantly suppressed IL-17A production in the spinal cord during inflammation. The data indicates that EA inhibits inflammatory pain by down-regulating spinal IL17A that facilitates pain.

Opioid receptor and nociceptin/orphanin FQ (N/OFQ) peptide receptor activation might mediate EA inhibition of IL-17A. Substance P (SP), a key neuropeptide for the transmission of noxious inputs, activates glial cells during pain [25]. SP release is blocked by $\mu$ and $\delta$ opioid receptor activation [26]. EA significantly activates these receptors [16] and has been shown to inhibit a tooth pulp stimulationevoked release of immunoreactive SP [27]. Opioid receptors are found in astrocytes [28], which suggests that opioids act directly on astrocytes to inhibit IL-17A synthesis. Taken together, these facts suggest that EA induces the release of endogenous spinal opioids that in turn inhibit cytokine synthesis in glial cells.

It has been reported that the opioid-receptor-like 1 (ORL1) are expressed on the spinal astrocytes in rats. Intrathecal administration of N/OFQ significantly downregulates CFA-injection-exaggerated spinal cord IL-1 $\beta$, IL-6, and TNF $\alpha$ expression in the inflammatory pain rat model [29]. Such inhibitory effect was abolished by the ORL1 receptor-specific antagonist [Nphe(1)]N/OFQ(1-13)NH2. In vitro studies with astrocyte cultures also show that $\mathrm{N} / \mathrm{OFQ}$ inhibits cytokine gene expression through the ORL1 receptor [29]. Since studies suggest that EA induces release of spinal N/OFQ [30, 31], it is plausible that EA-induced spinal N/OFQ might suppress spinal cytokine synthesis.

In this study, EA significantly mitigated CFA- and IL17A-enhanced NR1 phosphorylation in the spinal dorsal horn. It is well known that p-NR1 modulates NMDAR activity and facilitates nociceptive input transmission in inflammatory pain models [3-5]. This suggests that inflammation-enhanced IL-17A might facilitate pain through NR1 phosphorylation, and EA might decrease spinal
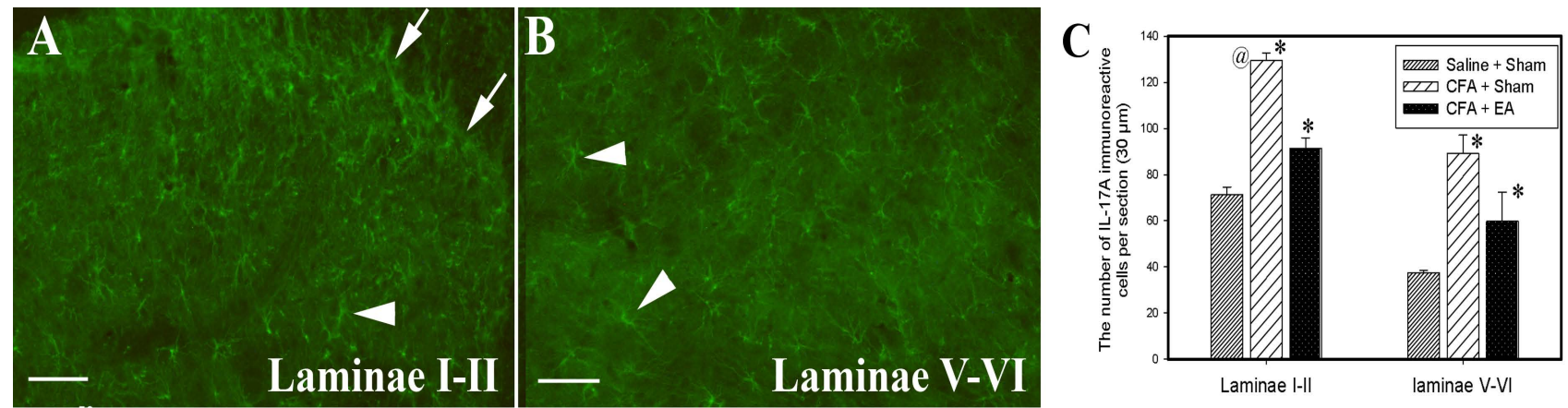

Fig. (2). IL-17A immunoreactive cell values in the spinal cord (mean \pm SEM). A-B: Representative photographs showing IL-17A immunoreactive cells in superficial laminae I-II (A) and V-VI (B) of the spinal cord $24 \mathrm{~h}$ after CFA + sham. Arrows point to superficial laminae. Arrow heads point to IL-17A immunoreactive cells. C: Note that the CFA injection induced more IL-17A than did a saline injection, and EA significantly inhibited IL-17A in laminae I-II compared to cham control *P $<.05$ vs. saline-injected rats; @P $<.05$ vs. CFA + EA. Scale bars $=50 \mu \mathrm{m}$. 


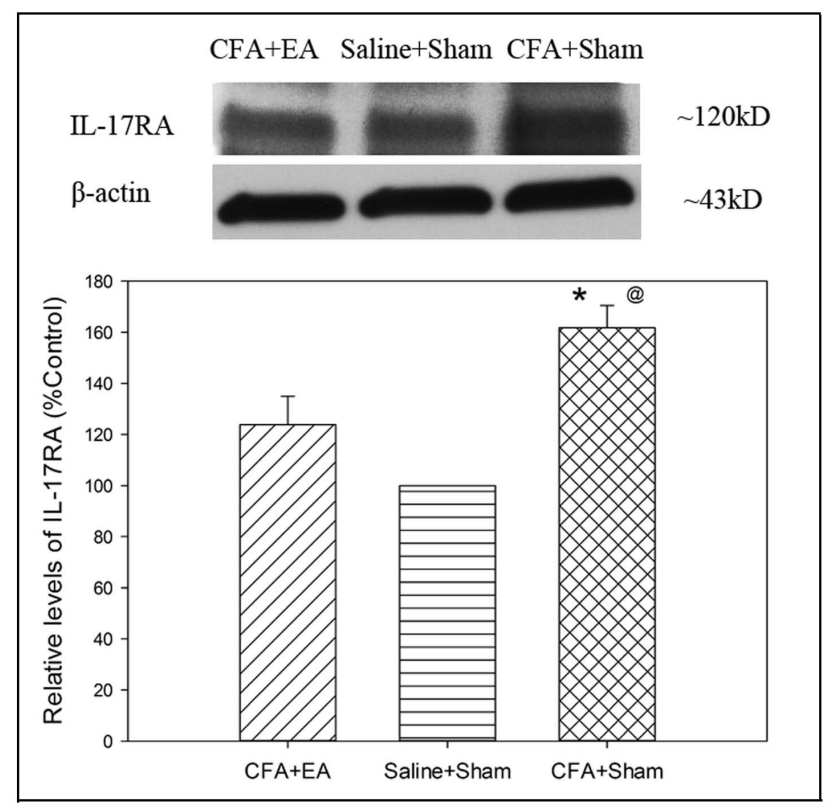

Fig. (3). Representative Western blot (upper panel) and quantification (lower panel) of relative IL-17RA levels in CFAinjected rats, which was normalized to saline-injected control $(100 \%)$. Note that IL-17RA level is higher in CFA- than in salineinjected rats and that EA treatment significantly decreased IL-RA expression compared to sham control. $* \mathrm{P}<.05$ vs. saline-injected control and $@ \mathrm{P}<.05$ vs. CFA + EA $(\mathrm{N}=4 /$ group $)$.

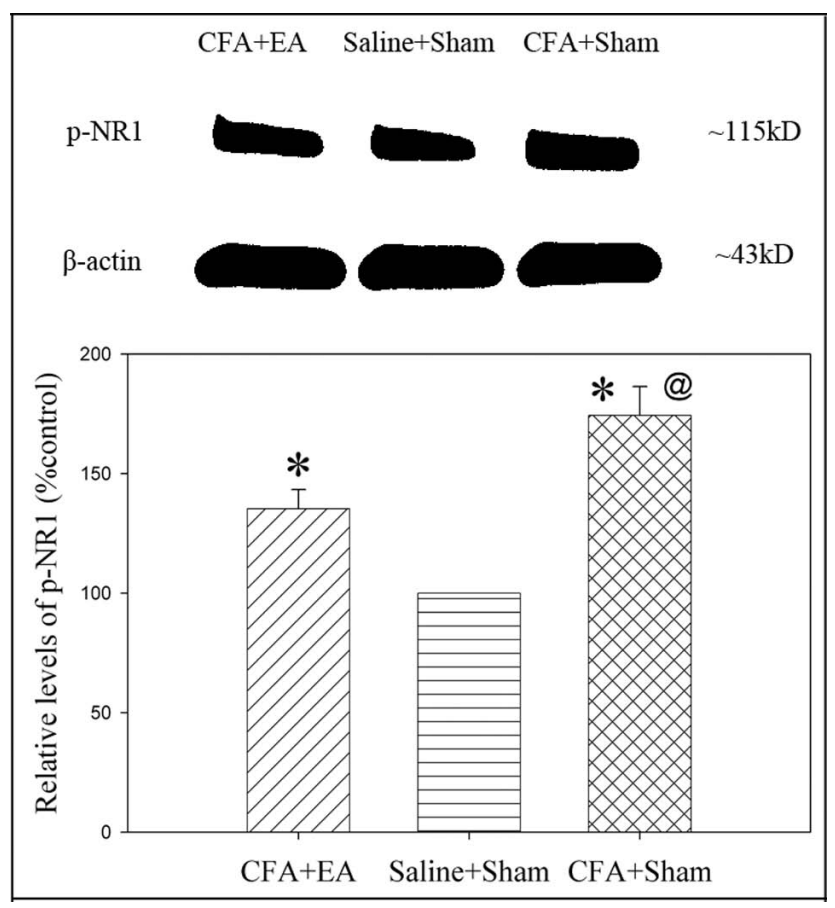

Fig. (5). Representative Western blot (upper panel) and quantification (lower panel) of relative p-NR1 levels in CFA-injected rats; saline-injected rats were used as control $(100 \%)$. Note that pNR1 level is higher in CFA rats than in control and that EA significantly decreased $\mathrm{p}-\mathrm{NR} 1$ compared to sham control. $* \mathrm{P}<.05$ vs. saline-injected control and $@ P<.05$ vs. CFA + EA $(\mathrm{N}=4 /$ group).

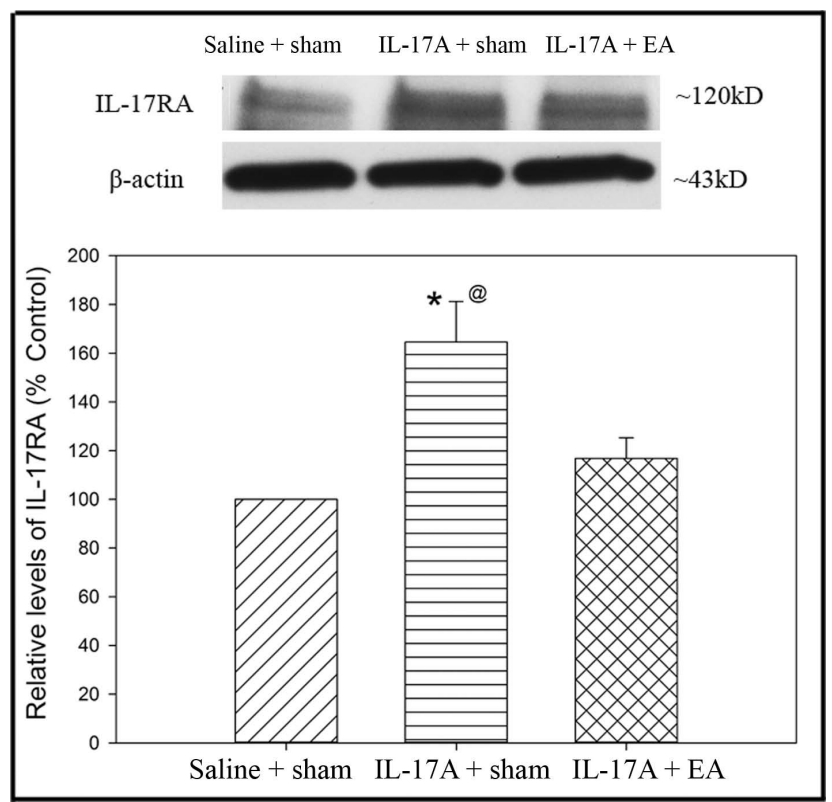

Fig. (4). Representative Western blot (upper panel) and quantification (lower panel) of relative IL-17RA levels in IL-17Ainjected rats, which was normalized to saline-injected control $(100 \%)$. Note that IL-17RA level is higher in IL-17A- than in salineinjected rats and that EA treatment significantly decreased IL-RA expression compared to sham control. ${ }^{*} \mathrm{P}<.05$ vs. saline-injected control and $@ \mathrm{P}<.05$ vs. IL-17A + EA $(\mathrm{N}=4$ /group $)$.

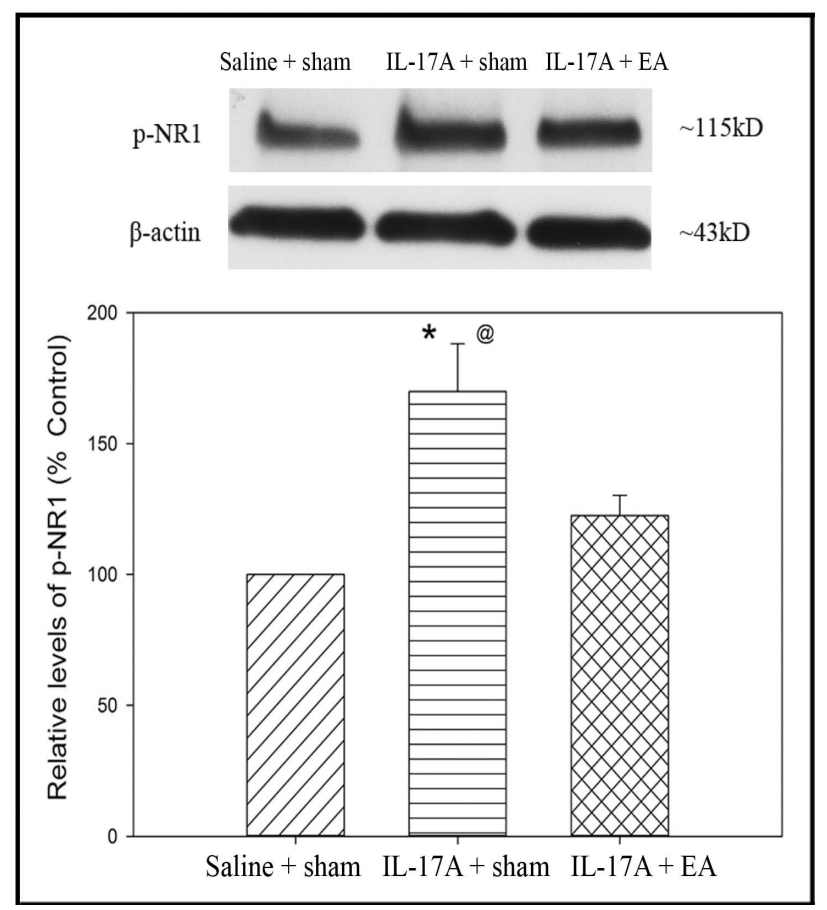

Fig. (6). Representative Western blot (upper panel) and quantification (lower panel) of relative p-NR1 levels in IL-17Ainjected rats; saline-injected rats were used as control (100\%). Note that $\mathrm{p}-\mathrm{NR} 1$ level is higher in IL-17A rats than in control and that EA significantly decreased $\mathrm{p}-\mathrm{NR} 1$ compared to sham control. $* \mathrm{P}<.05$ vs. saline-injected control and $@ P<.05$ vs. IL-17A + EA $(\mathrm{N}=4 /$ group). 
IL-17A, relieving the pain by diminishing NR1 phosphorylation. A previous study demonstrated that EA inhibits bone cancer-caused IL-1 $\beta$ expression and bone cancer pain [24]. IL-1 $\beta$ also promotes NR1 phosphorylation [6]. Together, these findings suggest that EA decreases noxious input-induced NR1 phosphorylation at the spinal level to alleviate pain.

We further showed that EA down-regulates inflammation- and IL-17A-induced IL-17RA, which may be associated to EA inhibition of NR1 phosphorylation. Previous studies demonstrate that IL-17RAs are localized in NR1-containing spinal neurons [9], and IL-17A antibodies block CFA- and IL-17A-caused IL-17RA up-regulation and NR1 phosphorylation [9]. Those data suggest that endogenous IL-17A may up-regulate IL-17RA on NR1containing neurons to facilitate NR1 phosphorylation. Thus EA down-regulation of inflammation-induced IL-17RA contributes to EA inhibition of NR1 phosphorylation.

A recent study showed that neuronal IL-17RA mediates IL-17A-induced up-regulation of transient receptor potential vanilloid 4 (TRPV4) in DRG neurons, suggesting that IL$17 \mathrm{~A}$ is involved in mechanical hyperalgesia [32]. It has been reported that EA increased mechanical threshold in the CFAinduced inflammatory pain model [33]. EA may modulate IL-17RA in DRG neurons to inhibit pain, which warrants further investigation.

\section{CONCLUSION}

The present study demonstrates that EA attenuates inflammatory hyperalgesia and inhibits astrocyte IL-17A, neuronal IL-17RA synthesis, and NR1 phosphorylation. The data suggest that EA alleviates pain by modulating glianeuron interaction and that IL-17A, IL-17RA, and NR1 phosphorylation are involved.

\section{CONFLICT OF INTEREST}

The author(s) confirm that this article content has no conflicts of interest.

\section{ACKNOWLEDGEMENT}

This publication was made possible by grant number R21AT005474-01 and P01AT002605 from the National Center for Complementary and Alternative Medicine (NCCAM) at the National Institutes of Health. The authors do not have any conflicts of interest. We would like to thank Dr. Lyn Lowry for her editorial support.

\section{ABBREVIATIONS}

$$
\begin{array}{ll}
\text { CFA } & =\text { Complete Freund's adjuvant } \\
\mathrm{EA} & =\text { Electroacupuncture } \\
\mathrm{IL}-1 \beta & =\text { Interleukin } 1 \text { beta } \\
\mathrm{IL}-6 & =\text { Interleukin } 6 \\
\mathrm{IL}-17 \mathrm{~A} & =\text { Interleukin-17A } \\
\mathrm{IL}-17 \mathrm{RA} & =\text { Interleukin-17 receptor A } \\
\mathrm{NMDAR} & =\text { N-methyl D-aspartate receptor } \\
\mathrm{N} / \mathrm{OFQ} & =\text { Nociceptin/orphanin FQ }
\end{array}
$$

ORL1 = Opioid-receptor-like 1

PWL = Paw withdrawal latency

TNF- $\alpha=$ Tumor necrosis factor alpha

TRPV4 = Transient receptor potential vanilloid 4

\section{REFERENCES}

[1] Watkins LR, Milligan ED, Maier SF. Glial proinflammatory cytokines mediate exaggerated pain states: implications for clinical pain. Adv Exp Med Biol 2003; 521: 1-21.

[2] Raghavendra V, Tanga FY, DeLeo JA. Complete Freunds adjuvant-induced peripheral inflammation evokes glial activation and proinflammatory cytokine expression in the CNS. Eur J Neurosci 2004; 20: 467-73.

[3] Zou X, Lin Q, Willis WD. Enhanced phosphorylation of NMDA receptor 1 subunits in spinal cord dorsal horn and spinothalamic tract neurons after intradermal injection of capsaicin in rats. J Neurosci 2000; 20: 6989-97.

[4] Zhang X, Wu J, Lei Y, et al. Protein phosphatase modulates the phosphorylation of spinal cord NMDA receptors in rats following intradermal injection of capsaicin. Mol Brain Res 2005; 138: 26472.

[5] Gao X, Kim HK, Chung JM, et al. Enhancement of NMDA receptor phosphorylation of the spinal dorsal horn and nucleus gracilis neurons in neuropathic rats. Pain 2005; 116: 62-72.

[6] Zhang RX, Li A, Liu B, et al. IL-1ra alleviates inflammatory hyperalgesia through preventing phosphorylation of NMDA receptor NR-1 subunit in rats. Pain 2008; 135: 232-9.

[7] Tzartos JS, Friese MA, Craner MJ, et al. Interleukin-17 production in central nervous system-infiltrating $\mathrm{T}$ cells and glial cells is associated with active disease in multiple sclerosis. Am J Pathol 2008; 172: 146-55.

[8] Costigan M, Moss A, Latremoliere A, et al. T-cell infiltration and signaling in the adult dorsal spinal cord is a major contributor to neuropathic pain-like hypersensitivity. J Neurosci 2009; 29: 1441522.

[9] Meng X, Zhang Y, Lao L, et al. 2013. Spinal interleukin-17 promotes thermal hyperalgesia and NMDA NR1 phosphorylation in an inflammatory pain rat model. Pain 2013; 154: 294-305.

[10] Berman BM, Lao L, Langenberg P, et al. Effectiveness of Acupuncture as Adjunctive Therapy in Osteoarthritis of the Knee. Ann Intern Med 2004;141: 901-10.

[11] Vickers AJ, Cronin AM, Maschino AC, et al. Acupuncture for chronic pain: individual patient data meta-analysis. Arch Intern Med 2012; 172: 1444-53.

[12] Kim HN, Kim YR, Jang JY, et al. Electroacupuncture confers antinociceptive effects via Inhibition of glutamate transporter downregulation in complete freund's adjuvant-injected rats. Evid Based Complement Alternat Med 2012; 2012: 643973.

[13] Shan S, Qi-Liang M-Y, Hong C, et al. Is functional state of spinal microglia involved in the anti-allodynic and anti-hyperalgesic effects of electroacupuncture in rat model of monoarthritis? Neurobiol Dis 2007; 26: 558-68.

[14] Sun S, Cao H, Han M, et al. Evidence for suppression of electroacupuncture on spinal glial activation and behavioral hypersensitivity in a rat model of monoarthritis. Brain Res Bull 2008; 75: 83-93.

[15] Mi WL, Mao-Ying QL, Wang X, et al. Involvement of spinal neurotrophin-3 in electroacupuncture analgesia and inhibition of spinal glial activation in rat model of monoarthritis. J Pain 2011; 12: 974-84.

[16] Zhang RX, Lao L, Wang L, et al. Involvement of opioid receptors in electroacupuncture-produced anti-hyperalgesia in rats with peripheral inflammation. Brain Res 2004; 1020: $12-7$.

[17] Hargreaves K, Dubner R, Brown F, et al. A new and sensitive method for measuring thermal nociception in cutaneous hyperalgesia. Pain 1988; 32: 77-88.

[18] Goldman N, Chen M, Fujita T, et al. Adenosine A1 receptors mediate local anti-nociceptive effects of acupuncture. Nature Neurosci 2010; 13: 883-8.

[19] Lao L, Zhang R-X, Zhang G, et al. A parametric study of electroacupuncture on persistent hyperalgesia and Fos protein expression in rats. Brain Res 2004; 1020: 18-29. 
[20] Koo ST, Lim K S, Chung K, et al. Electroacupuncture-induced analgesia in a rat model of ankle sprain pain is mediated by spinal $\alpha$-adrenoceptors. Pain 2008; 135: 11-9.

[21] Yin CS, Jeong HS, Park HJ, et al. A proposed transpositional acupoint system in a mouse and rat model. Res Vet Sci 2008; 84: 159-65.

[22] Koo ST, Park YI, Lim KS, et al. Acupuncture analgesia in a new rat model of ankle sprain pain. Pain 2002; 99: 423-31.

[23] Zhao Z-Q. Neural mechanism underlying acupuncture analgesia. Prog Neurobiol 2008; 85: 355-75.

[24] Zhang RX, Li A, Liu B, et al. Electroacupuncture attenuates bone cancer pain and inhibits spinal interleukin-1 beta expression in a rat model. Anesth Analg 2007; 105: 1482-8.

[25] Watkins LR, Maier SF. Glia: a novel drug discovery target for clinical pain. Nat Rev Drug Discov 2003; 2: 973-85.

[26] Bourgoin S, Benoliel JJ, Collin E, et al. Opioidergic control of the spinal release of neuropeptides. Possible significance for the analgesic effects of opioids. Fundam Clin Pharmacol 1994; 8: 30721.

[27] Yonehara N, Sawada T, Matsuura H, et al. Influence of electroacupuncture on the release of substance $\mathrm{P}$ and the potential evoked by tooth pulp stimulation in the trigeminal nucleus caudalis of the rabbit. Neurosci Lett 1992; 142: 53-6.
[28] Stiene-Martin A, Knapp PE, Martin K, et al. Opioid system diversity in developing neurons, astroglia, and oligodendroglia in the subventricular zone and striatum: impact on gliogenesis in vivo. Glia 2001; 36: 78-88.

[29] Fu X, Zhu ZH, Wang YQ, et al. Regulation of proinflammatory cytokines gene expression by nociceptin/orphanin FQ in the spinal cord and the cultured astrocytes. Neuroscience 2007; 144: 275-85.

[30] Fu X, Wang Y-Q, Wang J, et al. Changes in expression of nociceptin/orphanin $\mathrm{FQ}$ and its receptor in spinal dorsal horn during electroacupuncture treatment for peripheral inflammatory pain in rats. Peptides 2007; 28: 1220-8.

[31] Fu X, Wang YQ, Wu GC. Involvement of nociceptin/orphanin FQ and its receptor in electroacupuncture-produced anti-hyperalgesia in rats with peripheral inflammation. Brain Res 2006; 1078: 212-8.

[32] von Banchet GS, Boettger MK, König C, et al. Neuronal IL-17 receptor upregulates TRPV4 but not TRPV1 receptors in DRG neurons and mediates mechanical but not thermal hyperalgesia. Mol Cell Neurosci 2012; 52C:152-60.

[33] Su TF, Zhao YQ, Zhang LH, et al. Electroacupuncture reduces the expression of proinflammatory cytokines in inflamed skin tissues through activation of cannabinoid CB2 receptors. Eur J Pain 2012; 16: 624-35.

(C) Meng et al.; Licensee Bentham Open.

This is an open access article licensed under the terms of the Creative Commons Attribution Non-Commercial License (http://creativecommons.org/licenses/by-nc/3.0/) which permits unrestricted, non-commercial use, distribution and reproduction in any medium, provided the work is properly cited. 